OPEN ACCESS

Edited by:

Joshua B. Gurtler,

Agricultural Research Service

(USDA), United States

Reviewed by:

Lisa Gorski,

Western Regional Research Center

(USDA-ARS), United States

Teresa M. Bergholz,

North Dakota State University,

United States

*Correspondence:

Dennis J. D'Amico

ddamico@uconn.edu

Specialty section:

This article was submitted to

Agro Food Safety,

a section of the journal

Frontiers in Sustainable

Food Systems

Received: 11 December 2017

Accepted: 08 January 2018

Published: 29 January 2018

Citation:

Brown SRB, Kozak SM and

D'Amico DJ (2018) Applications of

Edible Coatings Formulated

with Antimicrobials Inhibit

Listeria monocytogenes Growth on

Queso Fresco.

Front. Sustain. Food Syst. 2:1.

doi: 10.3389/fsufs.2018.00001

\section{Applications of Edible Coatings Formulated with Antimicrobials Inhibit Listeria monocytogenes Growth on Queso Fresco}

\author{
Stephanie R. B. Brown, Sarah M. Kozak and Dennis J. D'Amico* \\ Department of Animal Science, University of Connecticut, Storrs, CT, United States
}

Despite efforts to control Listeria monocytogenes in dairy processing environments, contamination and subsequent outbreaks of listeriosis continue to occur. The ability of L. monocytogenes to grow during refrigerated storage necessitates strategies to prevent contamination, reduce pathogen numbers, and limit growth during storage. The objective of this study was to determine the efficacy of edible antimicrobial coatings to control L. monocytogenes on Queso Fresco (QF) when applied before [precoated (PC)] or after [preinoculated $(\mathrm{PI})$ ] surface contamination. Coating solutions were formulated to contain $2 \%$ chitosan and either $5 \%$ hydrogen peroxide (HP), 5\% lauric arginate (LAE), $25 \%$ acidified calcium sulfate with lactic acid (ACSL), or combinations of $10 \%$ sodium caprylate (SC) with either LAE or ACSL. Fresh QF samples (25 g) were inoculated with L. monocytogenes at $\sim 4 \log \mathrm{CFU} / g$ prior to, or following, antimicrobial coating application. Cheeses were then vacuum packaged and stored at $7^{\circ} \mathrm{C}$ for 35 days with weekly enumeration of $L$. monocytogenes. Aside from ACSL and LAE $+S C$, there was no effect of coating application timing (PC vs. PI) on the change in L. monocytogenes counts over time. Chitosan coatings without additional antimicrobials were more effective than controls but did not inhibit $L$. monocytogenes growth beyond 7 days. Coatings containing HP at 5\% were equally effective when applied before or after $L$. monocytogenes inoculation, significantly reducing $L$. monocytogenes counts by more than $3 \log \mathrm{CFU} / g$ and inhibiting growth through 35 days of storage. Coatings formulated with ACSL at 25\% were more effective when applied to PI cheeses but neither application produced significant reductions in L. monocytogenes counts or inhibited growth. Although LAE coatings were more effective than ACSL, neither were more effective than chitosan coatings alone. The addition of SC to ACSL and LAE coatings enhanced their antimicrobial activity as ACSL + SC and LAE + SC coatings reduced $L$. monocytogenes counts by $>1 \log C F U / g$ after $24 \mathrm{~h}$ and were listeristatic through 28 and 35 days, respectively. The identification of listericidal and listeristatic edible antimicrobial coating applications that are effective when applied before or after contamination events identifies a new approach for the control of $L$. monocytogenes on fresh cheese.

Keywords: Listeria monocytogenes, chitosan, cheese, antimicrobials, queso fresco, coating 


\section{INTRODUCTION}

According to the Centers for Disease Control and Prevention's Foodborne Outbreak Online Database, there were 19 reported outbreaks of listeriosis linked to dairy products between 2003 and 2016 resulting in 144 illnesses, 108 hospitalizations, and 21 deaths. Eleven of these outbreaks were linked to cheese known to be produced from pasteurized milk, six of which were Mexican or Hispanic-style soft cheeses including Queso Fresco (QF) (Centers for Disease Control and Prevention, 2017). Microbial risk assessments of Listeria monocytogenes in ready-to-eat foods suggest that preventing contamination altogether, followed by preventing the occurrence of high levels of contamination at consumption, would have the greatest impact on reducing illness rates [Chen et al., 2003; Food and Agriculture Organization and World Health Organization (FAO/WHO, 2004); U.S. Food and Drug Administration et al., 2003]. The application of antimicrobial coatings can inactivate, extend the lag-phase, and reduce the growth rate and maximum populations of microorganisms (Quintavalla and Vicini, 2002; Coma et al., 2003). Coatings can also provide an extra physical barrier to potentially limit initial contamination (Duan et al., 2007; Chen et al., 2012).

Chitosan is a natural, nontoxic, biodegradable, cationic polysaccharide obtained by alkaline deacetylation of the nitrogenous polysaccharide, chitin. Chitosan films and coatings have demonstrated antimicrobial activity against both bacteria and fungi both intrinsically and as a carrier of various antimicrobials (Kong et al., 2010). Chitosan coatings and films have been used to inhibit the growth of L. monocytogenes in a variety of foods and the incorporation of antimicrobials (e.g., essential oils, enzymes, bioactive compounds, etc.), can further enhance their efficacy (Pranoto et al., 2005; Zivanovic et al., 2005; Duan et al., 2007; Beverlya et al., 2008; Jiang et al., 2011; Petrou et al., 2012; Guo et al., 2014; Shekarforoush et al., 2015; Paparella et al., 2016). Previous research has identified effective antimicrobial treatments for the control of L. monocytogenes in broth and whole milk including acidified calcium sulfate with lactic acid (ACSL), hydrogen peroxide (HP), lauric arginate (LAE), and sodium caprylate (SC) (Kozak et al., 2017, 2018a). However, application of these antimicrobials to the surface of QF as aqueous dips were comparably less effective in inactivating L. monocytogenes and inhibiting growth during storage (Kozak et al., 2018b). Incorporation of these antimicrobials into edible chitosan films may enhance their efficacy, present a protective barrier, and allow for the progressive release of antimicrobials during storage. Maintaining high concentrations at the food surface may help extend the lag phase or inhibit growth following initial inactivation (Oussalah et al., 2004; Fajardo et al., 2010).

Although the use of chitosan coatings to extend shelf life has been tested in various cheeses (Coma et al., 2003; Altieri et al., 2005; Gammariello et al., 2008; Cerqueira et al., 2010), research on the use of chitosan coatings alone or formulated with antimicrobial compounds to control pathogens on cheese is limited. Generalizing from previous research is also difficult because the antimicrobial activity of chitosan and other antimicrobials is influenced by $\mathrm{pH}$, bacterial strain, incubation temperature, and food matrix (Devlieghere et al., 2004; Fernandez-Saiz et al., 2010).
Therefore, the objective of this study was to determine the efficacy of antimicrobial chitosan coatings, applied before or after surface contamination, to control L. monocytogenes on QF during storage at $7^{\circ} \mathrm{C}$.

\section{MATERIALS AND METHODS}

\section{Bacterial Strains, Growth Conditions, and Inoculum Preparation}

A cocktail was prepared as previously described (Kozak et al., 2017) using eight L. monocytogenes strains associated with outbreaks linked to soft cheeses or isolated from cheese processing environments (Table 1). The cocktail was serially diluted in Butterfield's phosphate buffer (BPB), pelleted through centrifugation $\left(30 \mathrm{~min}, 4,000 \mathrm{~g}\right.$ at $4^{\circ} \mathrm{C}$ ) (Thermo Scientific Sorvall Legend $\mathrm{X} 1 \mathrm{R}$, ThermoFisher Scientific, Waltham, MA, USA), and resuspended in BPB to attain $\sim 7 \log \mathrm{CFU} / \mathrm{mL}$.

\section{Cheese Manufacture and Analysis}

Queso Fresco was manufactured in the University of Connecticut Creamery according to a standard protocol as previously described (Kozak et al., 2018b). Compositional targets including $\sim 52 \%$ moisture, $\sim 22 \%$ fat, $\sim 2.0 \%$ salt, $\sim 67 \%$ moisture in the nonfat substance (MNFS), $45 \%$ fat in dry matter (FDM), $4 \%$ salt in moisture (SM), and $\mathrm{pH}$ of $\sim 6.4$, were based on previous work (Kozak et al., 2018b). Cheese blocks were vacuum packaged (Ultravac 250, UltraSource LLC, Kansas City, MO, USA) and stored at $4^{\circ} \mathrm{C}$ prior to cutting into experimental units.

Physiochemical analysis was conducted on each independent batch of cheese, in duplicate, at the time of cutting including: $\mathrm{pH}$ (Potentiometric Method) (Hooi et al., 2004) (Accumet AB150 with microtip electrode, Fisher Scientific International Inc., Hampton, NH, USA), dry matter (DM) (Forced Draft Oven Method) (Hooi et al., 2004), fat (Babcock Method) (Hooi et al., 2004), and salt (Quanttab Chloride, Hach, Loveland, CO, USA). SM, MNFS, and FDM were determined using the following formulae:

$$
\begin{aligned}
& \% \mathrm{SM}=(\% \text { salt } /(100-\% \mathrm{DM})) \times 100 \\
& \% \mathrm{MNFS}=(100-\% \mathrm{DM} /(100-\% \text { fat })) \times 100 \\
& \% \mathrm{FDM}=(\% \text { fat } / \% \mathrm{DM}) \times 100 .
\end{aligned}
$$

TABLE 1 | Name, source, ribotype, and serotype information for Listeria monocytogenes strains used in cocktail.

\begin{tabular}{lllc}
\hline Strain name & Source information & Ribotype & Serotype \\
\hline F5069/ATCC & Milk-related outbreak & DUP-1044B & $4 \mathrm{~b}$ \\
51414 & & & \\
CWD 675-3 & Hispanic-style cheese-related outbreak & DUP-1053A & $1 \mathrm{a}$ \\
CWD 1567 & Hispanic-style cheese-related outbreak & DUP-1038B & $4 \mathrm{~b}$ \\
Scott A & Milk-related outbreak & DUP-1042B & $4 \mathrm{~b}$ \\
2012L-5323 & Ricotta salata cheese-related outbreak & NA & $1 / 2 \mathrm{a}$ \\
2014L-6025 & Hispanic-style cheese-related outbreak & NA & $1 / 2 \mathrm{~b}$ \\
DJD 1 & Washed-rind cheese-related outbreak & NA & NA \\
CWD 193-10 & Dairy plant food contact surface & DUP-1030B & NA \\
U5-2 & & & \\
\hline
\end{tabular}

NA, not available. 


\section{Cheese Sample Preparation and Inoculation}

For each trial, cheeses were cut into $25 \pm 2 \mathrm{~g}$ samples using sterile knives within $48 \mathrm{~h}$ of manufacture and randomly assigned to one of two groups: preinoculated (PI) or precoated (PC). For inoculation of cheese in both groups, $100 \mu \mathrm{L}$ of the L. monocytogenes suspension in BPB was spread over a single surface of the cheese sample with a sterile spreader in order to attain a target contamination level of $\sim 4 \log \mathrm{CFU} / \mathrm{g}$ of cheese. Inoculated cheese samples with or without antimicrobial coating were allowed a 30-min drying period in a biosafety cabinet to enable bacterial attachment.

\section{Coating Preparation and Application}

Antimicrobial treatments for incorporation in chitosan coatings were selected based on previous work (Kozak et al., 2017, 2018a,b) and included: 25\% ACSL, 5\% HP, 5\% LAE, 25\% ACSL + 10\% SC, and $5 \%$ LAE $+10 \%$ SC. Preparation and application of chitosan coating solutions were adapted from previously published methods (No et al., 2002; Chen et al., 2012). Briefly, low-molecularweight (LMW) chitosan (50-190 kDa; Sigma Aldrich, St. Louis, MO, USA) was added at $2 \% \mathrm{wt} / \mathrm{v}$ to sterile deionized water (SDW) acidified with acetic acid (AA) ( $\geq 99.5 \%$, Sigma-Aldrich, St. Louis, MO, USA) at $1 \%(\mathrm{v} / \mathrm{v})$. For coating solutions containing ACSL (Mionix Corporation, Scottsdale, AZ, USA), chitosan was added to $1 \%$ AA solution after the addition of ACSL (25\% v/v). SC $(10 \%, w t / v)$ (Sigma Aldrich) and chitosan were added to $1 \% \mathrm{AA}$ solution at the same time for LAE + SC and prior to the addition of ACSL for ACSL + SC coatings. Antimicrobials in liquid form including HP (30\%, Acros Organic, Pittsburgh, PA, USA), and LAE (Cytoguard LA2X, A + B Ingredients, Fairfield, NJ, USA) were added directly to mixed chitosan solutions on a v/v basis. Coating solutions were adjusted to $\mathrm{pH} 4.5 \pm 0.05$ with AA or $10 \mathrm{~N} \mathrm{NaOH}$ and mixed overnight in an orbital shaker at room temperature before use.

Antimicrobial coatings were applied before or after inoculation with L. monocytogenes for PC and PI groups, respectively. Cheese samples (uninoculated for the PC group or inoculated for the PI group) were submerged in SDW (control), 1\% AA solution (AA control), 2\% chitosan coating solution (chitosan), or the respective antimicrobial coating solution for $1 \mathrm{~min}$ at room temperature. Samples were then removed using sterile tongs, placed onto a sterile drying rack, and the coating was evenly distributed across the exposed cheese surfaces with a sterile spreader. Samples were allowed to dry in a biosafety cabinet for up to $30 \mathrm{~min}$ to ensure a dry coating surface (Duan et al., 2007). After drying, samples in the PC group were inoculated with the L. monocytogenes cocktail as previously described. Inoculated and coated samples were placed in pouches ( 3 mil, oxygen transmission rate: $50-70 \mathrm{~cm}^{3} / \mathrm{m}^{2} 24 \mathrm{~h}$ ) (UltraSource LLC), vacuum-sealed (Ultravac 250 , UltraSource LLC), and stored at $7^{\circ} \mathrm{C}$ to mimic retail storage and mild temperature abuse along the food supply chain.

\section{Sampling and Enumeration}

Duplicate samples from each treatment and control were removed from storage on days 1, 7, 14, 21, 28, and 35 postinoculation. Samples were homogenized in $100 \mathrm{~mL}$ of Dey-Engley broth (DE)
(BD Difco, Becton, Dickinson and Company, Sparks, MD, USA) in a Smasher stomacher (Biomerieux, Marcy-l'Étoile, France) for $1 \mathrm{~min}$ at 560 strokes/min to neutralize antimicrobials. Following serial dilutions in $\mathrm{BPB}$, homogenates were plated onto modified Oxford agar (MOX) (BD Difco), incubated at $37^{\circ} \mathrm{C}$ for $48 \mathrm{~h}$, and enumerated. Two inoculated, uncoated samples were processed after the bacterial attachment period to verify initial inoculation levels. When applicable, $1 \mathrm{~mL}$ of the homogenate was plated over four MOX plates $(250 \mu \mathrm{L}$ per plate) to achieve a limit of detection (LOD) of $\geq 5 \mathrm{CFU} / \mathrm{g}$. Two uninoculated negative controls were also processed to verify the absence of $L$. monocytogenes in uninoculated QF based on this enumeration procedure.

\section{Data Analysis}

A completely randomized design with $15 \times 6$ factorial treatment structure (15 treatments at 6 time points) was followed. Experiments were performed in triplicate using three independently produced batches of cheese from three different days. Fresh working antimicrobial stock solutions were prepared for each biological replicate. Two cheese samples (technical replicates) were included for each treatment/time point/trial. Counts below the $\mathrm{LOD}$ of $\geq 5 \mathrm{CFU} / \mathrm{g}$ were recorded as $2.5 \mathrm{CFU} / \mathrm{g}(\mathrm{LOD} / 2)$ and counts from technical replicates were averaged for each trial $(n=3)$. One trial of HP (PI- and PC-HP) was removed from analysis after the stock solution used in coatings was found to be expired. PC-AA treatment also consists of only two biological replicates. Mean bacterial counts were log transformed and pooled data were analyzed using the PROC GLM procedure of SAS (ver. 9.4, SAS Institute, Cary, NC, USA). The model included treatment and time as main effects as well as treatment $\times$ time interactions. Pairwise comparisons were performed using LSMEANS with the Tukey or Tukey-Kramer method. Significance was defined as $P<0.05$. Inhibitory and bactericidal activity were defined as $<1 \log$ CFU/g increase and $\geq 3 \log$ CFU/g reduction in L. monocytogenes counts at a given time point compared to the initial inoculum level, respectively [National Advisory Committee on Microbiological Criteria for Foods (NACMCF, 2010); US Food and Drug Administration (FDA, 2017)].

\section{RESULTS AND DISCUSSION}

The physicochemical properties of the experimental QF batches were within target ranges (Table 2). Previous studies have estimated the shelf life of QF of similar composition packaged

TABLE 2 | Composition of Queso Fresco.

\begin{tabular}{lr}
\hline & Mean \pm SEM \\
\hline Dry matter & $44.4 \pm 1.3 \%$ \\
Moisture & $55.6 \pm 1.3 \%$ \\
Fat & $18.6 \pm 0.2 \%$ \\
Salt & $2.0 \pm 0.0 \%$ \\
MNFS & $68.3 \pm 1.5 \%$ \\
SM & $3.7 \pm 0.1 \%$ \\
FDM & $41.9 \pm 0.8 \%$ \\
pH & $6.44 \pm 0.01$
\end{tabular}

MNFS, moisture in the nonfat substance; SM, salt in moisture; FDM, fat in dry matter. 
under vacuum to be between 14 and 21 days (Brown et al. under review $)^{1}$. Enumeration of untreated controls on day 0 verified an initial inoculation level of $4.1 \pm 0.2 \mathrm{CFU} / \mathrm{g}$. No listeriae were

${ }^{1}$ Brown, S. R. B., Forauer, E. C., and D’Amico, D. J. (2018). Effect of modified atmosphere packaging on the growth of spoilage microorganisms and Listeria monocytogenes on fresh cheese. Under review. detected in uninoculated samples from each of the three batches of cheese. After a $24 \mathrm{~h}$ lag, L. monocytogenes grew rapidly on the surface of QF without antimicrobial application reaching counts $>8 \log \mathrm{CFU} / \mathrm{g}$ at day 21 with no additional change through 35 days (Figures 1 and 2), which is in agreement with previous studies (Soni et al., 2010; Kozak et al., 2018b). Treatment and time

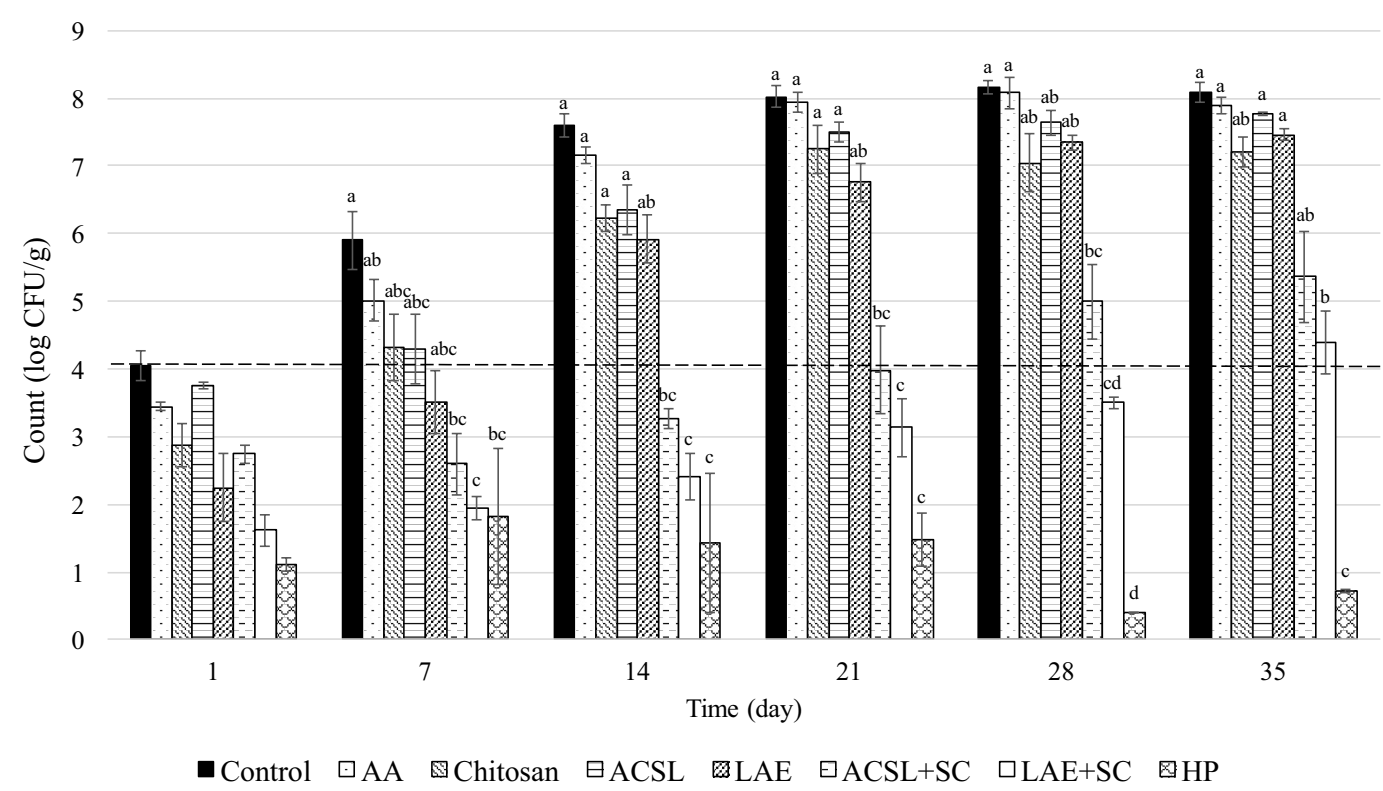

FIGURE 1 | Change in Listeria monocytogenes counts (mean \pm SEM) on Queso Fresco treated with single and combinatory antimicrobial coatings after inoculation (preinoculated). Dashed line indicates initial inoculation level. AA, acetic acid control (1\%); ACSL, acidified calcium sulfate with lactic acid (25\%); HP, hydrogen peroxide (5\%); LAE, lauric arginate (5\%); SC, sodium caprylate (10\%). Columns with different superscripts within a time point differ $(P<0.05)$.

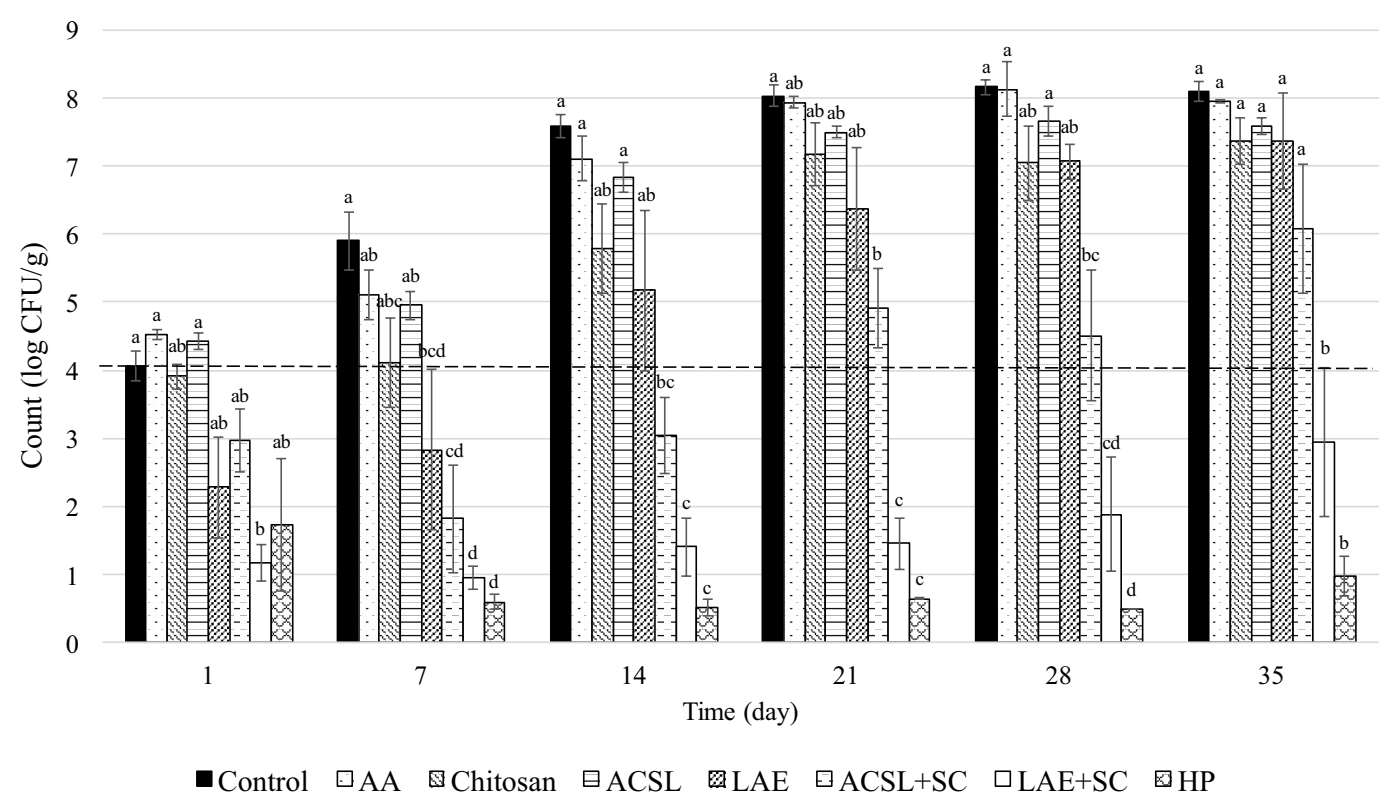

FIGURE 2 | Change in Listeria monocytogenes counts (mean \pm SEM) on Queso Fresco coated with single and combinatory antimicrobial coatings prior to inoculation (precoated). Dashed line indicates initial inoculation level. The control is the same control as Figure 1. AA, acetic acid control (1\%); ACSL, acidified calcium sulfate with lactic acid (25\%); HP, hydrogen peroxide (5\%); LAE, lauric arginate (5\%); SC, sodium caprylate (10\%). Columns with different superscripts within a time point differ $(P<0.05)$. 
effects as well as treatment $\times$ time interaction were observed for both PI and PC applications when treatments and controls were compared altogether $(P<0.001)$. Overall, the surface application of $1 \%$ AA to PI cheese (PI-AA control) had a significant effect on L. monocytogenes counts when compared to SDW control $(P=0.004)$ (Figure 1). The same effect was not observed for PC-AA control cheeses $(P=0.22)$ (Figure 2$)$, which suggests that the antimicrobial effect of AA may rely on more immediate contact with the acid or that acidity decreases following application to cheese and is less effective at the time of subsequent contamination.

\section{Antimicrobial Activity of Chitosan Coating}

Chitosan is typically applied to foods as either coatings or films. Coatings may be more suitable for the application to cheese to ensure direct contact in the presence of irregular surfaces (Guo et al., 2014). Chitosan is also thought to be more available as an antimicrobial in a coating solution compared to when it is used to form films (Zivanovic et al., 2005; Vásconez et al., 2009). The application of chitosan coatings to PI cheese in the present study (PI-chitosan) resulted in a $1.2 \mathrm{log} \mathrm{CFU} / \mathrm{g}$ reduction in mean L. monocytogenes counts at day 1 of sampling (Figure 1). This reduction was twice that of the PI-AA control $(0.6 \log \mathrm{CFU} / \mathrm{g})$ suggesting that the initial antimicrobial effect of chitosan coatings is not solely attributed to the presence of AA in the formulation. Counts on PI-chitosan treated cheeses returned to inoculation levels at day 7 and reached final population levels of $\sim 7 \log \mathrm{CFU} / \mathrm{g}$ on day 21 (Figure 1). Similar reductions $(\sim 1 \log C F U / g)$ in $L$. monocytogenes counts on Mozzarella cheese were also reported $24 \mathrm{~h}$ after application of chitosan coatings. Because untreated Mozzarella did not support the growth of L. monocytogenes during 14-day refrigerated storage, no subsequent growth was observed on chitosan-coated cheeses (Duan et al., 2007). Coma et al. (2003) reported substantial reductions in L. innocua after 3 days of storage following application of chitosan to Emmental cheese, but samples were stored at $37^{\circ} \mathrm{C}$ as opposed to lower temperatures more representative of typical refrigerated storage conditions. This is important considering lower temperatures may reduce chitosan efficacy due to reductions in the number of surface binding sites or electronegativity (Tsai and Su, 1999).

In contrast to coating PI cheese, the precoating of $\mathrm{QF}$ with chitosan (PC-chitosan) produced minimal reductions at day 1 with counts (3.9 log CFU/g) similar to initial inoculation levels (4.1 log CFU/g) and the PC-AA control (4 log CFU/g) (Figure 2). This may be explained in the same way as AA controls where initial antimicrobial activity relies on initial contact with the AA or chitosan in the coating or both. In the absence of diffusion, only organisms in direct contact with the active sites of chitosan are inhibited (Coma et al., 2003). It is possible that preapplication of chitosan coatings limits interaction between microorganisms with active sites compared to direct application of a liquid coating. Longer exposure to the components of the food matrix may also have inhibited the antimicrobial activity of chitosan. While the influence of fat may be negligible, protein and $\mathrm{NaCl}$ may inhibit the antimicrobial activity of chitosan as a result of competition for the positive charges on chitosan and the negative charges on the cell surfaces of the bacteria (Devlieghere et al., 2004). However, aside from differences in initial reductions after $24 \mathrm{~h}$, counts were similar between treatments (PI vs. PC) from day 7 onward and no treatment effects were observed between PC and PI-chitosan treatments. Significant treatment effects were observed for both PI- and PC-chitosan treatments when compared to SDW control $(P<0.001)$ and their corresponding PI- and PC-AA controls ( $P<0.001$ and $P=0.005$, respectively) (Figures 1 and 2).

\section{Antimicrobial Activity of Chitosan Coating with HP}

According to the Standards of Identity for cheese, HP is approved for use in certain varieties of cheese in the United States (i.e., cheddar, colby, washed or soaked curd, granular or stirred curd, swiss, and emmentaler) when added to milk at levels up to $500 \mathrm{ppm}$ followed by neutralization with catalase (21 CFR 133) [U.S. Food and Drug Administration (FDA), 2016]. HP has been shown to reduce L. monocytogenes counts and inhibit subsequent growth during storage when added to whole milk or applied as an aqueous dip to QF (Kozak et al., 2018a,b). In agreement with these reports, chitosan coatings formulated with $\mathrm{HP}$ at $5 \%$ were effective listericidal and listeristatic treatments (Figures 1 and 2). Aside from a single treatment (PC-LAE $+\mathrm{SC})$, there were significant treatment $\times$ time interactions when either PI- or PC-HP treatments were compared to all other treatments and controls. Application of HP coating to inoculated cheeses (PI-HP) reduced mean L. monocytogenes counts by $\geq 3 \log \mathrm{CFU} / \mathrm{g}$ at day 1 and counts were $<1 \log \mathrm{CFU} / \mathrm{g}$ at days 28 and 35 (Figure 1). Mean counts on cheeses PC with HP coatings (PC-HP) were $<1 \log \mathrm{CFU} / g$ at day 7 where they remained through 35 days of storage. Despite the lower counts observed with the PC-HP treatment, there was no effect of coating application timing (PI-HP vs. PC-HP) on L. monocytogenes counts.

\section{Antimicrobial Activity of Chitosan Coating with ACSL}

Acidified calcium sulfate with lactic acid is generally recognized as safe (GRAS) and suitable for use in several animal food products [U.S. Department of Agriculture, Food Safety and Inspection Service (USDA, 2009)]. Application of ACSL as an aqueous dip (25\%) on QF reduced L. monocytogenes counts after $24 \mathrm{~h}$, but growth was not inhibited throughout the remaining storage period (Kozak et al., 2018b). Because coatings have been shown to maintain high concentrations of antimicrobials at the food surface through their progressive release during storage (Oussalah et al., 2004; Fajardo et al., 2010), the incorporation ACSL into chitosan coatings could potentially limit growth following initial reductions. However, the application of chitosan coatings formulated with ACSL at $25 \%$ yielded minimal changes in L. monocytogenes counts by day 1 in both the PI- and PC-ACSL treatments and both treatments were only inhibitory through day 7 (4.3 and 4.4 $\log$ CFU/g for PI- and PC-ACSL, respectively) (Figures 1 and 2). Although there was a significant effect of treatment type (PI vs. PC) with lower counts on PI-ACSL cheeses compared to PC-ACSL $(P=0.046)$, counts on cheeses for both treatments were lower than SDW control $(P<0.001)$ and their corresponding AA controls $(P<0.001$ and $P=0.022$ for PI and $P C$, respectively). In 
contrast, both treatments resulted in counts on cheeses that were higher than chitosan coating alone $(P=0.04$ and $P=0.011$ for PI-ACSL and PC-ACSL, respectively), suggesting that the addition of ACSL at $25 \%$ does not enhance the antimicrobial activity of chitosan coatings.

Overall, the efficacy of ACSL incorporated into chitosan coatings appears to be less than that observed when applied to QF as an aqueous dip, which produced initial reductions of $1.4 \mathrm{log}$ CFU/g after $24 \mathrm{~h}$ (Kozak et al., 2018b). Mean counts reported for ACSL dip applications were more than one log lower than those on ACSL-coated cheeses in the present study through 14 days (5.2 $\log$ CFU/g) (Kozak et al., 2018b). Mean counts on both the PIand PC-ACSL treated cheeses also exceeded $7 \log$ CFU/g at day 21, which was one week earlier than reported for dip application (Kozak et al., 2018b). Because the mechanism of action for ACSL is attributed to the dissociation and reprotonation of lactic acid (Kemp et al., 2003), buffering the $\mathrm{pH}$ of the coating ( $\mathrm{pH} \mathrm{4.5)} \mathrm{in}$ the present study could have contributed to a loss of antimicrobial activity.

\section{Antimicrobial Activity of Chitosan Coating with LAE}

Similar to ACSL, LAE has been shown to produce initial inactivation of L. monocytogenes on the surface of QF when applied as an aqueous dip followed by subsequent growth (Kozak et al., 2018b). The application of coating solutions formulated with LAE at 5\% reduced mean L. monocytogenes counts on QF by 1.7 and 1.8 $\log$ CFU/g when applied before (PC-LAE) or after inoculation (PI-LAE), respectively (Figures 1 and 2). Despite a difference in counts of $1.2 \log \mathrm{CFU} / \mathrm{g}$ at day 7, there was no effect of coating application timing (PI-LAE vs. PC-LAE) on L. monocytogenes counts. Significant treatment effects were observed when both PIand PC-LAE cheeses were compared to SDW control $(P<0.001)$ and their corresponding AA controls $(P<0.001$ and $P=0.003$ for PI and PC, respectively). PI-LAE and PC-LAE were also more effective in reducing $L$. monocytogenes counts when compared to cheeses coated with ACSL $(P<0.001$ and $P=0.002$, respectively), which is in contrast with results reported for aqueous dip application of these antimicrobials (Kozak et al., 2018b). Similar to ACSL coatings, L. monocytogenes counts on both the PI- and PC-LAE cheeses did not differ from PI or PC-chitosan treatments suggesting that the addition of LAE at $5 \%$ does not enhance the antimicrobial activity of chitosan coatings. L. monocytogenes counts following LAE application as an aqueous dip at 5\% to surface contaminated QF (Kozak et al., 2018b) were also similar to those observed in the present study, suggesting that formulating LAE in a chitosan-based antimicrobial coating may not enhance its antimicrobial effects.

Guo et al. (2014) demonstrated that antimicrobial coating treatments with $1.94 \mathrm{mg} / \mathrm{cm}^{2}$ chitosan ( 2\% LMW chitosan) and $0.388 \mathrm{mg} / \mathrm{cm}^{2} \mathrm{LAE}$ reduced L. innocua counts by $\sim 4.6 \mathrm{log} \mathrm{CFU} /$ $\mathrm{cm}^{2}$ on turkey deli meat after $24 \mathrm{~h}$ at $10^{\circ} \mathrm{C}$ (Guo et al., 2014). Although this is a significant reduction compared to those observed in the present study, it is not known whether L. innocua counts increased during subsequent storage. However, chitosan coating treatments formulated with LAE ( $\sim \% \mathrm{LAE})$ reduced initial counts and inhibited the growth of L. monocytogenes on roast beef throughout the 30 -day storage period at $4^{\circ} \mathrm{C}$ when inoculated at 2.8 and $5.3 \log \mathrm{CFU} / \mathrm{cm}^{2}$ (Wang et al., 2015). Chitosan coatings containing 1\% LAE also reduced Salmonella populations on egg shells by $5.6 \log$ units at day 1 and then to undetectable levels on day 3 during storage at $7^{\circ} \mathrm{C}$ (Jin et al., 2013). In addition to differences in $\mathrm{pH}$, bacterial species, incubation temperature, and food matrices (Devlieghere et al., 2004; Fernandez-Saiz et al., 2010), the differences in antimicrobial activity between studies could be attributed to type of acid used to produce coating solutions. Previous studies have demonstrated that the combination of lactic, acetic, and levulinic acids used in the formulation of these coating solutions has antimicrobial activity against Listeria and Salmonella in various foods (Chen et al., 2012; Jin and Gurtler, 2012).

It is possible that higher concentrations of LAE would be more effective in the control of L. monocytogenes on QF. However, LAE is only considered GRAS for use as an antimicrobial agent in cheeses (e.g., curd and whey cheeses, cream, natural, grating, processed, spread, and dip cheeses) at levels up to $200 \mathrm{ppm}$ [U.S. Food and Drug Administration (FDA), 2005]. The impact of higher concentrations of LAE on the sensory properties of cheese is also not known. LAE can bind to anionic biopolymers naturally present within the mouth leading to a perceived bitterness or astringency, but the impact of complexation interactions (i.e., LAE and chitosan) on the sensory attributes of LAE (i.e., ability to cause astringency) are also not known (Bonnaud et al., 2010). As an alternative, the use of combinations of antimicrobials that work additively or synergistically to inhibit or inactivate L. monocytogenes can potentially enhance antimicrobial efficacy without increasing individual usage concentrations (Kozak et al., 2017).

\section{Antimicrobial Activity of Chitosan Coating with SC in Combination with ACSL or LAE}

Submerging QF in $10 \%$ solutions of SC inhibited L. monocytogenes growth to $<1 \log \mathrm{CFU} / g$ through 21 days of storage (Kozak et al., 2018b). Addition of SC to LAE dip treatments also inhibited $L$. monocytogenes growth on QF through 21 days and worked synergistically with ACSL to inhibit L. monocytogenes counts on QF through 28 days of storage (Kozak et al., 2018b). Based on these findings, coatings were formulated to include ACSL and LAE in combination with SC to limit growth during storage. Caprylic acid, but not SC, is currently considered GRAS at levels not to exceed current good manufacturing practices, which result in maximum levels, as served, of $400 \mathrm{ppm}$ for cheeses and $50 \mathrm{ppm}$ for frozen dairy desserts [U.S. Food and Drug Administration (FDA), 2017a]. Application of ACSL + SC coating to inoculated cheese (PI-ACSL + SC) reduced mean L. monocytogenes counts by 1.3 and $1.5 \log \mathrm{CFU} / \mathrm{g}$ at days 1 and 7 , respectively (Figure 1 ). Counts eventually returned to initial inoculation levels at day 21 but the treatment was listeristatic through day 28 with counts reaching $5 \log \mathrm{CFU} / \mathrm{g}$. Significant time $\times$ treatment interactions were observed when counts on PI-ACSL + SC coated cheeses were compared to SDW control $(P=0.009)$, PI-AA control $(P=0.002)$, PI-chitosan $(P=0.018)$, and PI-LAE $(P=0.005)$. Counts were also lower than PI-ACSL coating alone $(P=0.045)$ (Figure 1). The efficacy of PC-ASCL + SC was similar to that of 
PI-ACSL + SC. Mean L. monocytogenes counts were reduced by $1.1 \log$ CFU/g by the day 1 sampling point and reached a low of 1.8 $\log \mathrm{CFU} / \mathrm{g}$ on day 7. Counts increased thereafter but the treatment was listeristatic through 28 days (Figure 2). Treatment $\times$ time interactions were observed when counts on PC-ACSL + SC treated cheeses were compared to SDW control $(P=0.037)$ and treatment effects were seen when compared to PC-AA control $(P<0.001)$, PC-chitosan $(P<0.001)$, PC-LAE $(P=0.011)$, and PC-ACSL $(P<0.001)$ (Figure 2$)$. As with ACSL coatings, the application of ACSL + SC coatings were less effective against L. monocytogenes on QF when compared with aqueous dip applications (Kozak et al., 2018b).

Aside from PI-HP, PI-LAE + SC was more effective than all other treatments and controls with treatment $\times$ time interactions observed when compared to SDW control $(P<0.001)$, PI-AA control $(P<0.001)$, PI-chitosan $(P=0.003)$, PI-ACSL $(P=0.003)$, and PI-LAE $(P<0.001)$ (Figure 1). Though no significant interaction was observed, counts were lower than PI-ACSL + SC $(P<0.001)$ as well. At $24 \mathrm{~h}$, mean counts were reduced to $1.6 \mathrm{log}$ $\mathrm{CFU} / \mathrm{g}$ and the treatment was listeristatic through 35 days with a final count of $4.4 \log \mathrm{CFU} / \mathrm{g}$ on day 35 of storage (Figure 1).

Precoating cheese with LAE + SC reduced mean L. monocytogenes counts by $2.9 \mathrm{log} \mathrm{CFU} / \mathrm{g}$ at $24 \mathrm{~h}$ and counts gradually increased during storage to $3 \mathrm{log} \mathrm{CFU} / \mathrm{g}$ at 35 days (Figure 2). There was a significant treatment effect for LAE + SC applications whereby this combinatory antimicrobial coating was more effective in reducing $L$. monocytogenes counts on QF when applied before (PC-LAE + SC) than after inoculation (PI-LAE + SC) $(P<0.001)$. Treatment $\times$ time interactions were observed when the change in L. monocytogenes counts on PC-LAE + SC treated cheeses were compared to SDW control $(P=0.007)$, PC-ACSL $(P=0.038)$, and PC-LAE $(P<0.001)$. No significant interaction was observed but counts were lower than PC-AA control $(P<0.001)$, PC-chitosan $(P<0.001)$, PC-LAE $(P<0.001)$, and $\mathrm{PC}-\mathrm{ACSL}+\mathrm{SC}(P<0.001)$ (Figure 2). In contrast to ACSL + SC coatings, counts at 35 days for both PI- and PC-LAE + SC were lower than those reported for LAE + SC application as an aqueous dip, which only inhibited growth through 28 days reaching $4.7 \log$ CFU/g at day 35 (Kozak et al., 2018b).

The addition of SC in the formulation of ACSL + SC solutions increased the $\mathrm{pH}$ to 4.5 , which was similar to that of chitosan alone. When SC was added to the LAE coating solution the $\mathrm{pH}$ increased to $\sim 7.25$. Because higher $\mathrm{pH}$ levels impede the solubility of chitosan and for consistency between treatments, the $\mathrm{pH}$ of $\mathrm{LAE}+\mathrm{SC}$ coating solutions was reduced to 4.5 using glacial AA. Synergistic activity between organic acids and chitosan (Rhoades and Roller, 2000) in addition to any antimicrobial effects of SC alone may help explain the enhanced efficacy of this treatment compared to LAE alone. Because the antimicrobial efficacy of chitosan is enhanced with increased acidity (Wang, 1992; No et al., 2002; Duan et al., 2007; Kong et al., 2010), future studies are needed to determine the efficacy of coatings formulated at a lower $\mathrm{pH}$.

Although a formal sensory evaluation was outside the scope of the present study, chitosan coatings can bind water and lipids and may therefore serve as a protective barrier against moisture loss and delay lipid oxidation (Sathivel, 2005; Vásconez et al.,
2009). Previous studies on chitosan application to chicken breast (Petrou et al., 2012), cold-smoked salmon (Jiang et al., 2011), and turkey meat (Vardaka et al., 2016) suggest that chitosan may not negatively influence the sensory characteristics of these products. Studies on the effects of antimicrobials alone or formulated in chitosan coatings or films on the sensory properties of foods are limited. Sensory panels evaluating medium-well roast beef samples coated with a chitosan solution formulated with LAE were able to identify the difference between treated and control samples immediately after surface coating treatments but not on day 15 (Wang et al., 2015). Similarly, Otero et al. (2014) reported that polypropylene and polyethylene tetrephalate films coated with LAE did not significantly affect the organoleptic properties of Zamorano cheese slices. Therefore, chitosan solutions formulated with LAE may not negatively influence the organoleptic properties of cheese, but future studies are warranted.

In addition to those studies previously discussed, antimicrobial coatings and films formulated with compounds other than chitosan have also been investigated for the control of Listeria on cheeses with some similar results. For example, application of a nisin-incorporated sodium caseinate film resulted in a $\sim 1.1$ $\log \mathrm{CFU} / \mathrm{g}$ reduction in L. innocua counts in surface-inoculated processed cheese samples after 1 week of storage at $4^{\circ} \mathrm{C}$ as compared to control samples (Cao-Hoang et al., 2010). Similarly, chitosan-grafted lactic acid packaging reduced $L$. monocytogenes populations on fresh cheese by $\sim 1 \log \mathrm{CFU} / \mathrm{g}$ by day 7 at $4^{\circ} \mathrm{C}$ (Sandoval et al., 2016). Zein (protein from maize) and zeincarnauba wax composite films formulated with lysozyme were effective in suppressing the growth of L. monocytogenes in fresh cheeses (Kashar) during cold-storage with significant reductions in L. monocytogenes counts occurring when films were formulated with sustained lysozyme-release (Ünalan et al., 2013).

The focus of this study was to determine the efficacy of chitosan-based antimicrobial coatings applied to the surface of QF before or after surface contamination to control L. monocytogenes growth during cold storage. Efficacy of treatments was based on current FDA guidance whereby a listeristatic formulation that combines one or more antimicrobial substances is generally considered to be effective as process controls if growth studies show an increase of $<1 \log$ cycle over two or more time intervals during product shelf life [U.S. Food and Drug Administration (FDA), 2017b]. The present study identifies effective listericidal and listeristatic controls. Coatings containing HP at 5\% were equally effective when applied before or after L. monocytogenes inoculation, significantly reducing L. monocytogenes counts by more than $3 \mathrm{log}$ CFU/g and inhibiting growth through 35 days of storage. Coatings formulated with ACSL at 25\% were more effective when applied to PI cheeses but neither application produced significant reductions in L. monocytogenes counts or inhibited growth. The application of coating solutions formulated with LAE at 5\% reduced mean L. monocytogenes counts on the QF by 1.7-1.8 log CFU/g whether applied before or after inoculation. Although LAE coatings were more effective than those containing ACSL, neither were more effective than chitosan coatings alone, suggesting that the addition of LAE at $5 \%$ or ACSL at $25 \%$ does not enhance the antimicrobial activity of chitosan coatings applied before or after L. monocytogenes 
contamination. The addition of SC to ACSL and LAE coatings enhanced their effectiveness. ACSL + SC coatings reduced L. monocytogenes counts by 1.1-1.3 log CFU/g after $24 \mathrm{~h}$ and were listeristatic through 28 days. LAE + SC treatments were more effective in reducing $L$. monocytogenes counts on QF when applied before inoculation than after, but both treatments produced $>1 \log \mathrm{CFU} / \mathrm{g}$ reductions at $24 \mathrm{~h}$ and were listeristatic through 35 days.

This study identifies a novel approach to control L. monocytogenes. Applications of edible antimicrobial coatings containing $\mathrm{HP}$ or SC in combination with ACSL or LAE were effective in controlling postlethality L. monocytogenes contamination of $\mathrm{QF}$. Effective coating formulations not only inactivate and inhibit L. monocytogenes already present on the surface of cheese, but provide a protective barrier to proliferation when applied before contamination events. Future studies are needed to evaluate potential changes to the sensory properties of QF and similar fresh and soft cheeses containing antimicrobials at effective concentrations to ensure consumer acceptance.

\section{DATA AVAILABILITY}

Datasets analyzed for this study supporting the conclusions of this manuscript will be made available by the authors, without undue reservation, to any qualified researcher.

\section{REFERENCES}

Altieri, C., Scrocco, C., Sinigaglia, M., and Del Nobile, M. A. (2005). Use of chitosan to prolong mozzarella cheese shelf life. J. Dairy Sci. 88, 2683-2688. doi:10.3168/ jds.S0022-0302(05)72946-5

Beverlya, R. L., Janes, M. E., Prinyawiwatkula, W., and No, H. K. (2008). Edible chitosan films on ready-to-eat roast beef for the control of Listeria monocytogenes. Food Microbiol. 25, 534-537. doi:10.1016/j.fm.2007.11.002

Bonnaud, M., Weiss, J., and McClements, D. J. (2010). Interaction of a food-grade cationic surfactant (lauric arginate) with food-grade biopolymers (pectin, carrageenan, xanthan, alginate, dextran, and chitosan). J. Agric. Food Chem. 58, 9770-9777. doi:10.1021/jf101309h

Cao-Hoang, L., Chaine, A., Grégoire, L., and Waché, Y. (2010). Potential of nisin-incorporated sodium caseinate films to control Listeria in artificially contaminated cheese. Food Microbiol. 27, 940-944. doi:10.1016/j.fm.2010. 05.025

Centers for Disease Control and Prevention. (2017). Foodborne Outbreak Online Database (FOOD Tool). Available at: https://wwwn.cdc.gov/ foodborneoutbreaks/

Cerqueira, M. A., Sousa-Gallagher, M. J., Macedo, I., Rodriguez-Aguilera, R., Souza, B. W., Teixeira, J. A., et al. (2010). Use of galactomannan edible coating application and storage temperature for prolonging shelf-life of "Regional" cheese. J. Food Eng. 97, 87-94. doi:10.1016/j.jfoodeng.2009.09.019

Chen, W., Jin, T., Gurtler, J. B., Geveke, D. J., and Fan, X. (2012). Inactivation of Salmonella on whole cantaloupe by application of an antimicrobial coating containing chitosan and Allyl Isothiocyanate. Int. J. Food Microbiol. 155, 165-170. doi:10.1016/j.ijfoodmicro.2012.02.001

Chen, Y., Ross, W. H., Scott, V.N., and Gombas, D. E. (2003). Listeria monocytogenes: low levels equal low risk. J. Food Prot. 66, 570-577. doi:10.4315/0362-028X66.4 .570

Coma, V., Deschamps, A., and Martial-Gros, A. (2003). Bioactive packaging materials from edible chitosan polymer-antimicrobial activity assessment on dairy-related contaminants. J. Food Sci. 68, 2788-2792. doi:10.111 1/j.1365-2621.2003.tb05806.x

Devlieghere, F., Vermeulen, A., and Debevere, J. (2004). Chitosan: antimicrobial activity, interactions with food components and applicability as a coating on fruit and vegetables. Food Microbiol. 21, 703-714. doi:10.1016/j.fm.2004.02.008

\section{AUTHOR CONTRIBUTIONS}

DD conceived and designed the experiments. SK and SB performed the experiments. SB performed the statistical analysis. $\mathrm{SB}$ and DD wrote sections of the manuscript. DD revised the manuscript. All authors read and approved the submitted version.

\section{ACKNOWLEDGMENTS}

The authors would like to thank the Mionix Corporation and A + B Ingredients for supplying antimicrobials for use in this study. The sponsors and companies supplying materials had no involvement in study design, the collection, analysis and interpretation of data, or in the writing of this manuscript.

\section{FUNDING}

This project was supported by the National Dairy Council (award \#02368) and the United States Department of Agriculture (USDA) National Institute of Food and Agriculture (NIFA), Multistate project S1056. Any opinions, findings, conclusions, or recommendations expressed in this manuscript are those of the author(s) and do not necessarily reflect the view of the NIFA, the USDA or any other sponsors.

Duan, J., Park, S. I., Daeschel, M. A., and Zhao, Y. (2007). Antimicrobial chitosanlysozyme (CL) films and coatings for enhancing microbial safety of mozzarella cheese. J. Food Sci. 72, M355-M362. doi:10.1111/j.1750-3841.2007.00556.x

Fajardo, P., Martins, J. T., Fuciños, C., Pastrana, L., Teixeira, J. A., and Vicente, A. A. (2010). Evaluation of a chitosan-based edible film as carrier of natamycin to improve the storability of Saloio cheese. J. Food Eng. 101,349-356. doi:10.1016/j. jfoodeng.2010.06.029

Fernandez-Saiz, P., Soler, C., Lagaron, J. M., and Ocio, M. J. (2010). Effects of chitosan films on the growth of Listeria monocytogenes, Staphylococcus aureus and Salmonella spp. in laboratory media and in fish soup. Int. J. Food Microbiol. 137, 287-294. doi:10.1016/j.ijfoodmicro.2009.11.016

Food and Agriculture Organization, and World Health Organization (FAO/ WHO). (2004). Risk Assessment of Listeria monocytogenes in Ready-to-Eat Foods. Technical Report. Available at: http://www.fao.org/3/a-y5394e.pdf

Gammariello, D., Chillo, S., Mastromatteo, M., Di Giulio, S., Attanasio, M., and Del Nobile, M. A. (2008). Effect of chitosan on the rheological and sensorial characteristics of Apulia Spreadable Cheese. J. Dairy Sci. 91, 4155-4163. doi:10.3168/jds.2008-1280

Guo, M., Jin, T. Z., Wang, L., Scullen, O. J., and Sommers, C. H. (2014). Antimicrobial films and coatings for inactivation of Listeria innocua on ready-to-eat deli turkey meat. Food Control 40, 64-70. doi:10.1016/j.foodcont.2013.11.018

Hooi, R., Barbano, D. M., Bradley, R. L., Budde, D., Bulthaus, M., Chettiar, M., et al. (2004). "Chemical and physical methods," in Standard Methods for the Examination of Dairy Products, 17th Edn, eds H. M. Wehr and J. F. Frank (Washington, DC: American Public Health Association), 363-536.

Jiang, Z., Neetoo, H., and Chen, H. (2011). Control of Listeria monocytogenes on cold-smoked salmon using chitosan-based antimicrobial coatings and films. J. Food Sci. 76, M22-M26. doi:10.1111/j.1750-3841.2010.01925.x

Jin, T. Z., and Gurtler, J. B. (2012). Inactivation of Salmonella on tomato stem scars by edible chitosan and organic acid coatings. J. Food Prot. 75, 1368-1372. doi:10.4315/0362-028X.JFP-12-054

Jin, T. Z., Gurtler, J. B., and Li, S. Q. (2013). Development of antimicrobial coatings for improving the microbiological safety and quality of shell eggs. J. Food Prot 76, 779-785. doi:10.4315/0362-028X.JFP-12-460

Kemp, M. C., Lalum, R. B., Lewis, D. E., Carpenter, R. H., and Mionix Corporation. (2003). Highly Acidic Metalated Organic Acid as a Food Additive. U.S. Patent $6,572,908$. Washington, DC: U.S. Patent and Trademark Office. 
Kong, M., Chen, X. G., Xing, K., and Park, H. J. (2010). Antimicrobial properties of chitosan and mode of action: a state of the art review. Int. J. Food Microbiol. 144, 51-63. doi:10.1016/j.ijfoodmicro.2010.09.012

Kozak, S. M., Brown, S. R. B., Bobak, Y., and D’Amico, D. J. (2018a). Control of Listeria monocytogenes in whole milk using antimicrobials applied individually and in combination. J. Dairy Sci. doi:10.3168/jds.2017-13648

Kozak, S. M., Bobak, Y., and D'Amico, D. J. (2018b). Efficacy of antimicrobials applied individually and in combination for controlling Listeria monocytogenes as surface contaminants on Queso Fresco. J. Food Prot. 81, 46-53. doi:10.4315/0362-028X.JFP-17-279

Kozak, S. M., Margison, K. M., and D’Amico, D. J. (2017). Synergistic antimicrobial combinations inhibit and inactivate Listeria monocytogenes in neutral and acidic broth systems. J. Food Prot. 80, 1266-1272. doi:10.4315/0362-028X.JFP17-035

National Advisory Committee on Microbiological Criteria for Foods (NACMCF). (2010). Parameters for determining inoculated pack/challenge study protocols. J. Food Prot. 73, 140-202. doi:10.4315/0362-028X-73.1.140

No, H. K., Park, N. Y., Lee, S. H., and Meyers, S. P. (2002). Antibacterial activity of chitosans and chitosan oligomers with different molecular weights. Int. J. Food Microbiol. 74, 65-72. doi:10.1016/S0168-1605(01)00717-6

Otero, V., Becerril, R., Santos, J. A., Rodríguez-Calleja, J. M., Nerín, C., and García-López, M. L. (2014). Evaluation of two antimicrobial packaging films against Escherichia coli O157: H7 strains in vitro and during storage of a Spanish ripened sheep cheese (Zamorano). Food Control 42, 296-302. doi:10.1016/j. foodcont.2014.02.022

Oussalah, M., Caillet, S., Salmiéri, S., Saucier, L., and Lacroix, M. (2004). Antimicrobial and antioxidant effects of milk protein-based film containing essential oils for the preservation of whole beef muscle. J. Agric. Food Chem. 52, 5598-5605. doi:10.1021/jf049389q

Paparella, A., Mazzarrino, G., Chaves-López, C., Rossi, C., Sacchetti, G., Guerrieri, O., et al. (2016). Chitosan boosts the antimicrobial activity of Origanum vulgare essential oil in modified atmosphere packaged pork. Food Microbiol. 59, 23-31. doi:10.1016/j.fm.2016.05.007

Petrou, S., Tsiraki, M., Giatrakou, V., and Savvaidis, I. N. (2012). Chitosan dipping or oregano oil treatments, singly or combined on modified atmosphere packaged chicken breast meat. Int. J. Food Microbiol. 156, 264-271. doi:10.1016/j. ijfoodmicro.2012.04.002

Pranoto, Y., Rakshit, S. K., and Salokhe, V. M. (2005). Enhancing antimicrobial activity of chitosan films by incorporating garlic oil, potassium sorbate and nisin. LWT-Food Sci. Technol. 38, 859-865. doi:10.1016/j.lwt.2004.09.014

Quintavalla, S., and Vicini, L. (2002). Antimicrobial food packaging in meat industry. Meat Sci. 62, 373-380. doi:10.1016/S0309-1740(02)00121-3

Rhoades, J., and Roller, S. (2000). Antimicrobial actions of degraded and native chitosan against spoilage organisms in laboratory media and foods. Appl. Environ. Microbiol. 66, 80-84. doi:10.1128/AEM.66.1.80-86.2000

Sandoval, L. N., López, M., Montes-Díaz, E., Espadín, A., Tecante, A., Gimeno, M., et al. (2016). Inhibition of Listeria monocytogenes in fresh cheese using chitosan-grafted lactic acid packaging. Molecules 21, 469. doi:10.3390/ molecules21040469

Sathivel, S. (2005). Chitosan and protein coatings affect yield, moisture loss, and lipid oxidation of pink salmon (Oncorhynchus gorbuscha) fillets during frozen storage. J. Food Sci. 70, E455-E459. doi:10.1111/j.1365-2621.2005.tb11514.x

Shekarforoush, S. S., Basiri, S., Ebrahimnejad, H., and Hosseinzadeh, S. (2015). Effect of chitosan on spoilage bacteria, Escherichia coli and Listeria monocytogenes in cured chicken meat. Int. J. Biol. Macromol. 76, 303-309. doi:10.1016/j. ijbiomac.2015.02.033

Soni, K. A., Nannapaneni, R., Schilling, M. W., and Jackson, V. (2010). Bactericidal activity of lauric arginate in milk and queso fresco cheese against Listeria monocytogenes cold growth. J. Dairy Sci. 93, 4518-4525. doi:10.3168/jds.20103270

Tsai, G. J., and Su, W. H. (1999). Antibacterial activity of shrimp chitosan against Escherichia coli. J. Food Prot. 62, 239-243. doi:10.4315/0362-028X-62.3.239
Ünalan, İU., Arcan, İ, Korel, F., and Yemenicioğlu, A. (2013). Application of active zein-based films with controlled release properties to control Listeria monocytogenes growth and lipid oxidation in fresh Kashar cheese. Innov. Food Sci. Emerg. 20, 208-214. doi:10.1016/j.ifset.2013.08.004

U.S. Department of Agriculture, Food Safety and Inspection Service (USDA). (2009). Safe and Suitable Ingredients Used in the Production of Meat and Poultry Products. FSIS Directive 7120.1 Amendment 6. Available at: https://www.fsis.usda.gov/wps/ wcm/connect/bab10e09-aefa-483b-8be8-809a1f051d4c/7120.1.pdf?MOD= AJPERES

U.S. Food and Drug Administration (FDA). (2005). GRAS Notice No. GRN 000164. Available at: http://wayback.archive-it.org/7993/20171031052522/ https://www.fda.gov/downloads/Food/IngredientsPackagingLabeling/GRAS/ NoticeInventory/UCM268847.pdf

U.S. Food and Drug Administration (FDA). (2016). Code of Federal Regulations (CFR) Title 21. Available at: http://www.accessdata.fda.gov/scripts/cdrh/cfdocs/ cfCFR/CFRSearch.cfm

U.S. Food and Drug Administration (FDA). (2017). Draft Guidance for Industry: Control of Listeria monocytogenes in Ready-to-Eat Foods. Available at: https:// www.fda.gov/RegulatoryInformation/Guidances/ucm073110.htm

U.S. Food and Drug Administration (FDA). (2017a). "Chapter I, Subchapter B, Part 184," in Title 21: Code of Federal Regulations. Available at: https://www. accessdata.fda.gov/scripts/cdrh/cfdocs/cfcfr/CFRSearch.cfm?fr $=184.1025$

U.S. Food and Drug Administration (FDA). (2017b). Draft Guidance for Industry: Control of Listeria monocytogenes in Ready-to-Eat Foods. Available at: https:// www.fda.gov/RegulatoryInformation/Guidances/ucm073110.htm

U.S. Food and Drug Administration, Center for Food Safety and Applied Nutrition, and U.S. Department of Agriculture, Food Safety and Inspection Service (FDA/ USDA). (2003). Quantitative Risk Assessment of the Relative Risk to Public Health from Foodborne Listeria monocytogenes among Selected Categories of Ready-to-Eat Foods. Available at: https://www.fda.gov/downloads/Food/ FoodScienceResearch/UCM197330.pdf

Vardaka, V. D., Yehia, H. M., and Savvaidis, I. N. (2016). Effects of Citrox and chitosan on the survival of Escherichia coli O157: H7 and Salmonella enterica in vacuum-packaged turkey meat. Food Microbiol. 58, 128-134. doi:10.1016/j. fm.2016.04.003

Vásconez, M. B., Flores, S. K., Campos, C. A., Alvarado, J., and Gerschenson, L. N. (2009). Antimicrobial activity and physical properties of chitosan-tapioca starch based edible films and coatings. Food Res. Int. 42, 762-769. doi:10.1016/j. foodres.2009.02.026

Wang, G. H. (1992). Inhibition and inactivation of five species of foodborne pathogens by chitosan. J. Food Prot. 55, 916-919. doi:10.4315/0362-028X55.11 .916

Wang, L., Zhao, L., Yuan, J., and Jin, T. Z. (2015). Application of a novel antimicrobial coating on roast beef for inactivation and inhibition of Listeria monocytogenes during storage. Int. J Food Microbiol. 211, 66-72. doi:10.1016/j. ijfoodmicro.2015.07.007

Zivanovic, S., Chi, S., and Draughon, A. F. (2005). Antimicrobial activity of chitosan films enriched with essential oils. J. Food Sci. 70, M45-M51. doi:10.111 1/j.1365-2621.2005.tb09045.x

Conflict of Interest Statement: The authors declare that the research was conducted in the absence of any commercial or financial relationships that could be construed as a potential conflict of interest.

The reviewer LG and handling editor declared their shared affiliation.

Copyright (C) 2018 Brown, Kozak and D'Amico. This is an open-access article distributed under the terms of the Creative Commons Attribution License (CC BY). The use, distribution or reproduction in other forums is permitted, provided the original author(s) and the copyright owner are credited and that the original publication in this journal is cited, in accordance with accepted academic practice. No use, distribution or reproduction is permitted which does not comply with these terms. 